\title{
Author's reply: acute serologic autoimmune hepatitis in the post-partum period
}

\author{
Noriyuki Akutsu $^{1} \cdot$ Keisuke Ishigami $^{1} \cdot$ Hiroshi Nakase $^{1}$
}

Received: 11 March 2020 / Accepted: 12 March 2020 / Published online: 30 March 2020

(c) Japanese Society of Gastroenterology 2020

We appreciate Efe et al. s' interest in our case and read your case with great interest. Since the first description of autoimmune hepatitis (AIH) by Waldenstörm in the early 1950s [1], AIH was considered to be a chronic liver disease of unknown etiology with variable patterns of presentation. Over the last decades, AIH can occur as acute onset hepatitis or fulminant hepatic failure [2]. In $20-30 \%$ of cases, AIH causes jaundice and a subset of these patients go on to acute liver failure (ALF) or fulminant failure. This pattern has not been extensively described in the medical literature and represents a challenge for physicians.

In this case reported by Efe et al., a female patient diagnosed with ALF one month after delivery, who was diagnosed with AIH based on both her negative liver serology and the histological result of liver biopsy specimens, was successfully treated with prompt administration of corticosteroid. They suggested the importance of considering AIH as a differential diagnosis of ALF after baby delivery.

Generally, patients with AIH-induced ALF are frequently negative for serological markers including anti-nuclear, antismooth muscle, and anti-liver kidney antibodies. In addition, major histocompatibility complex HLA-loci (HLA-DR3 and HLA-DR4) are reported to have a strong association with AIH [3]. Interestingly, a recent study demonstrated that drugs, viral infections, and surgery in general anesthesia might trigger ALF as the initial presentation of AIH [4]. Thus, the genetic risk factor in combination with environmental factors, which are involved in the disruption of immune tolerance to the autologous liver antigens, leads to the onset of AIH. In this regard, we strongly suggest that Efe should show the detail medical history before the onset of ALF and HLA-DR to clarify the etiology of her AIH.

Noriyuki Akutsu

akutsu@ sapmed.ac.jp

1 Department of Gastroenterology and Hepatology, Sapporo Medical University School of Medicine, Minami 1-Jo Nishi 16-Chome, Chuo-ku, Sapporo, Hokkaido, Japan
Nevertheless, Efe's case is clinically important for thinking about the etiology of AIH.

Therefore, further investigation will be required to elucidate the exact mechanism of AIH after baby delivery by accumulating clinical cases.

\section{Compliance with ethical standards}

Conflicts of interest Noriyuki Akutsu, Keisuke Ishigami and Hiroshi Nakase declare that they have no conflict of interest.

Human rights All procedures followed have been performed in accordance with the ethical standards laid down in the 1964 Declaration of Helsinki and its later amendments.

Informed consent Informed consent was obtained from all patients for being included in the study.

\section{References}

1. Waldenstrom J. Leber. Blutproteine und Nahrungseiweiss. Dtsch Ges Verdau Stoffwechselkr. 1950;15:113-9.

2. Verma S, Maheshwari A, Thuluvath P. Liver failure as initial presentation of autoimmune hepatitis: clinical characteristics, predictors of response to steroid therapy, and outcomes. Hepatology. 2009;49:1396-7.

3. Strettell MD, Donaldson PT, Thomson LJ, et al. Allelic basis for HLA-encoded susceptibility to type 1 autoimmune hepatitis. Gastroenterology. 1997;112:2028-35.

4. Buechter M, Manka P, Heinemann FM, et al. Potential triggering factors of acute liver failure as a first manifestation of autoimmune hepatitis-a single center experience of 52 adult patients. World $\mathbf{J}$ Gastroenterol. 2018;24:1410-8.

Publisher's Note Springer Nature remains neutral with regard to jurisdictional claims in published maps and institutional affiliations. 